\title{
A randomised trial of Mycobacterium $w$ in critically ill patients with COVID-19: ARMY-1
}

\author{
Inderpaul Singh Sehgal (1) ${ }^{1}$, Randeep Guleria ${ }^{2}$, Sarman Singh ${ }^{3}$, \\ Mohammad Sabah Siddiqui ${ }^{4}$ and Ritesh Agarwal ${ }^{1}$, for the ARMY trial study group
}

Affiliations: ${ }^{1}$ Dept of Pulmonary Medicine, Postgraduate Institute of Medical Education and Research, Chandigarh, India. ${ }^{2}$ All India Institute of Medical Sciences, New Delhi, India. ${ }^{3}$ All India Institute of Medical Sciences, Bhopal, India. ${ }^{4}$ Dept of Medicine, All India Institute of Medical Sciences, Raipur, India.

Correspondence: Inderpaul Singh Sehgal, Dept of Pulmonary Medicine, Postgraduate Institute of Medical Education and Research, Sector-12, Chandigarh-160012, India. E-mail: inderpgiaoutlook.com

\section{ABSTRACT}

Purpose: We investigated whether Mycobacterium $w(M w)$, an immunomodulator, would improve clinical outcomes in coronavirus disease 2019 (COVID-19).

Methods: We conducted an exploratory, randomised, double-blind, placebo-controlled trial of hospitalised subjects with severe COVID-19 (pulmonary infiltrates and oxygen saturation $\leqslant 94 \%$ on room air) conducted at four tertiary care centres in India. Patients were randomised 1:1 to receive either $0.3 \mathrm{~mL} \cdot$ day $^{-1}$ of $\mathrm{Mw}$ intradermally or a matching placebo for three consecutive days. The primary outcome of the study was the distribution of clinical status assessed on a seven-point ordinal scale ranging from discharged (category 1 ) to death (category 7 ) on study days 14,21 , and 28 . The co-primary outcome was a change in SOFA (sequential organ failure assessment) score on days 7 and 14 compared to the baseline. The secondary outcomes were 28-day mortality, time to clinical recovery, time to reverse transcription PCR negativity, adverse events, and others.

Results: We included 42 subjects (22 Mw, 20 placebo). On days 14 (OR 30.4 (95\% CI 3.3-276.4)) and 21 (OR 14.9 (95\% CI 1.8-128.4)), subjects in the $M w$ arm had a better clinical status distribution than placebo. There was no difference in the SOFA score change on days 7 and 14 between the two groups. We did not find any difference in the mortality, or other secondary outcomes. We observed no adverse events related to the use of $M w$.

Conclusions: The use of $M w$ results in better clinical status distribution on days 14 and 21 compared to placebo in critically ill patients with COVID-19.

@ERSpublications

In this exploratory, multicentre, randomised, double-blind, placebo-controlled trial of 42 patients with severe \#COVID19, a significantly better distribution of clinical status on days 14 and 21 was found for those randomised to the Mycobacterium $w$ arm https://bit.ly/3q0sjOZ

Cite this article as: Sehgal IS, Guleria R, Singh S, et al. A randomised trial of Mycobacterium $w$ in critically ill patients with COVID-19: ARMY-1. ERJ Open Res 2021; 7: 00059-2021 [https://doi.org/ 10.1183/23120541.00059-2021].

This study is registered at www.clinicaltrials.gov with identifier number NCT04347174. We will share the data on request.

Received: 25 Jan 2021 | Accepted: 22 Feb 2021

Copyright $\odot$ The authors 2021. This version is distributed under the terms of the Creative Commons Attribution NonCommercial Licence 4.0. For commercial reproduction rights and permissions contact permissions@ersnet.org 


\section{Introduction}

Coronavirus disease 2019 (COVID-19) can cause illness ranging from asymptomatic cases to severe disease, including death. The organ damage in severe COVID-19 is believed to be due to a dysregulated host immune response [1-4]. In patients with severe COVID-19, there is a sustained reduction of peripheral lymphocytes, mainly CD4 and CD8 T-cells [5, 6]. Also, there is a suppression of type I and type III interferon leading to low viral clearance [2,7]. The use of immunosuppressive drugs, such as systemic corticosteroids and anti-interleukin (IL)-6 receptor monoclonal antibodies, further suppress the immunity [8-12]. Thus, patients with severe COVID-19 are likely to have a sustained immune-paralytic state after the initial pro-inflammatory state, similar to Gram-negative sepsis [3]. Interestingly, those who recover mount a T-helper (Th) 1 predominant response compared with the historical controls with non-COVID infection [13].

Mycobacterium w $(M w)$, also known as Mycobacterium indicus pranii, is a non-pathogenic, rapidly growing atypical mycobacterium. $M w$ shares T- and B-cell determinants with Mycobacterium leprae and Mycobacterium tuberculosis. Heat-killed $M w$ administered intradermally is a potent Toll-like receptor (TLR)-2 agonist [14], inhibits TLR-9 [15], and augments the Th1 immune response [16]. Mw enhances the expression of IL-1 receptor associated kinase- 1 and tumour necrosis factor receptor associated factor- 6 that are critical for activation of TLR-4 downstream kinases [17]. $M w$ also upregulates the inhibitor $\kappa$ kinase- $\alpha$ and $-\beta$ [17]. $M w$ has been studied for its immune-modulating properties in patients with pulmonary tuberculosis, tuberculous pericarditis, sepsis, lung cancer, and leprosy [17-22]. Elsewhere, $M w$ alone and in combination with anti-retroviral therapy increased the CD4 T-cell count in patients with HIV [23]. Previously, we have demonstrated that $M w$ (at a dose of $0.3 \mathrm{~mL}$ intradermally for 3 days) in combination with standard care, reduced the all-cause 28-day mortality (unpublished data), the days spent on mechanical ventilation, and the intensive care unit (ICU) and hospital length of stay [19]. We hypothesised that $M w$, by its immunomodulatory mechanism, would result in clinical improvement in severe acute respiratory syndrome-coronavirus-2 (SARS-CoV-2). Herein, we evaluate the role of $M w$ as adjunctive therapy to standard care in critically ill patients with COVID-19.

\section{Methods}

Study design

We conducted an exploratory, multicentre, randomised, double-blind, two parallel arm, comparative controlled trial (to evaluate the efficacy of $M w$ in combination with standard therapy in critically ill subjects with COVID-19. Each participating centre obtained ethical approval from the respective institutional ethics committee. We obtained written informed consent from all subjects or the next of kin. We conducted the trial according to the principles of the Declaration of Helsinki and the International Conference on Harmonisation Good Clinical Practice guidelines. The protocol is available at www. clinicaltrials.gov (identifier NCT04347174). No amendments were made to the protocol after commencement of the trial. The subjects were enrolled between 15 June 2020 and 15 July 2020, and the final follow-up visit was on 15 August 2020. The study product is marketed by Cadila Pharmaceuticals India and is a regulatory trial. However, the study was conducted under a public-private partnership through a grant received from the Council of Scientific and Industrial Research (Government of India) under the New Millennium Indian Technology Leadership Initiative (NMITLI). Cadila Pharmaceuticals provided the study drug and the matching placebo and was not involved in the data analysis or interpretation of the results.

\section{Setting}

The study was conducted in ICUs or high dependency units of four tertiary care centres in India.

\section{Patients}

We screened consecutive patients aged $>18$ years who were positive for SARS-CoV-2 RNA on reverse transcriptase (RT)-PCR. We included subjects with a saturation of $\leqslant 94 \%$ on ambient air and infiltrates on a chest radiograph. Subjects of childbearing age were included if they agreed to take effective contraception measures during the study period. We excluded subjects with any of the following: 1) pregnancy or breastfeeding; 2) prior cardiorespiratory arrest; 3) chronic liver disease; 4) haemodialysis dependent chronic kidney disease; 5) enrolment in another trial; 6) active malignancy; and 7) subjects unwilling to provide consent. The use of other drugs according to the institutional protocol at each participating centre was allowed. Briefly, the treatments included systemic glucocorticoids, hydroxychloroquine, convalescent plasma, tocilizumab, and anticoagulation. 


\section{Trial monitoring}

An independent data safety and monitoring board monitored the trial periodically and evaluated the study data for participant safety, study conduct, and progress. An independent steering committee oversaw the conduct of the entire study.

\section{Randomisation}

A central team not directly involved in patient care or patient data analysis provided a computer-generated randomisation sequence. The randomisation was stratified according to the centres. The subjects at each centre received either the investigational drug or a matched placebo in individually numbered packs according to the sequential order. The investigators at each participating centre and the subjects were blinded to the treatment allocation. Envelopes were provided to each participating centre for emergency unmasking.

\section{Sample size}

The initial target of the study was 40 subjects. The ARMY-1 trial was designed to assess the feasibility and safety of $M w$ in severe COVID-19. The results were presented to the regulatory body of India, namely the drug controller general of India (DCGI), to seek permission for the next phase of the trial (ARMY-2).

\section{Study procedures}

We recorded clinical data for all the subjects on paper case record forms that were subsequently entered into an electronic database and validated by the trial staff at each centre. We assessed the respiratory rate, oxygen supplementation device used (nasal cannula, venturi mask, non-rebreathing mask, high-flow nasal cannula, noninvasive ventilation, or invasive mechanical ventilation), oxygen saturation, the use of concomitant medications, and adverse events during hospitalisation. We performed complete blood count, blood glucose, liver and renal function tests, arterial blood gas analysis each day until day 7 , and then on days 14, 21 and 28, if the patient was still hospitalised. We recorded the sequential organ failure assessment (SOFA) score for each day until hospital discharge. We obtained nasopharyngeal or oropharyngeal or endotracheal aspirate for detecting COVID-19 RNA by RT-PCR at days 5, 7, 14, 21, 28 , or at the time of hospital discharge.

We evaluated the clinical status of the study participants from day 1 through day 28 or hospital discharge on a seven-point ordinal scale consisting of the following: category 1: not hospitalised with the resumption of normal activities; category 2: not hospitalised but unable to resume normal activities; category 3 : hospitalised but not requiring supplemental oxygen; category 4: hospitalised and requiring supplemental oxygen (nasal cannula, venturi mask, or non-rebreathing mask); category 5: hospitalised and requiring nasal high-flow oxygen therapy, noninvasive mechanical ventilation, or both; category 6: hospitalised and requiring invasive mechanical ventilation or extracorporeal membrane oxygenation (ECMO); and category 7: death. We recorded the worst score for clinical status every day for hospitalised subjects. We made the final assessment on day 28 in person for a hospitalised subject or telephonically for those discharged before day 28 .

\section{Study drug}

Each dose of $0.1 \mathrm{~mL} M w$ contains $0.5 \times 10^{9}$ heat killed Mycobacterium w, $0.9 \%$ sodium chloride, and $0.01 \%$ thimerosal (as preservative). We used a matching placebo $(0.9 \%$ sodium chloride, $0.01 \%$ thiomersal $)$ as control.

\section{Intervention}

Subjects were randomised to receive a single daily dose of $0.3 \mathrm{~mL} M w$ or a matching placebo (in aliquots of $0.1 \mathrm{~mL}$ at three different sites) intradermally in the deltoid region for three consecutive days. We administered the study drugs within $24 \mathrm{~h}$ of admission to the hospital. We observed the subjects for any adverse effects, local or systemic, that could be associated with the administration of the study drug.

\section{Study outcomes}

The primary outcome of the study was the distribution of clinical status assessed on the seven-point ordinal scale on days 14, 21 and 28 after randomisation. If treatment with $M w$ improved the outcomes, the distribution of the scores among patients who received $M w$ would shift more towards lower values of the scale than the distribution of the scores among patients who received a placebo. The co-primary outcome was a change in SOFA score (delta SOFA) on days 7 and 14 compared to the baseline and the maximum SOFA attained during the hospital stay.

The secondary outcomes were 28-day mortality, the proportion of patients with adverse events that occurred on or after the first dose of the study drug for up to 28 days, time to clinical recovery (defined as 
a reduction by two-points on a seven-point ordinal scale), time to one-point improvement on a seven-point ordinal scale, days on vasopressor drugs, days on mechanical ventilation, time to RT-PCR negativity, and ICU and hospital length of stay.

\section{Statistical analysis}

We present the data descriptively as mean \pm SD, median (first and third quartile), or number (\%). The difference between the continuous and categorical variables was analysed using the Mann-Whitney U-test (or student's t-test) and Chi-squared test, respectively. We performed all analyses on an intention-to-treat basis. All the subjects who were randomised and received at least one study dose were assessed for efficacy and safety. If the subject died before day 14, the day 14 category on the ordinal scale was recorded as "died"; if the subject was discharged before day 14, the category on day 14 was recorded as "not hospitalised". Similarly, if the subject died before day 7 or day 14 , we assumed the highest value for the SOFA (score of 20) or the value just before death on days 7 and 14. We used the proportional odds model for the ordinal scale data, including treatment as the independent variable and the baseline disease severity, and the use of experimental therapies (hydroxychloroquine, convalescent plasma and tocilizumab) as covariates. An odds ratio $>1$ would suggest treatment with $M w$ to be superior to placebo on days 14, 21 and 28. We assumed statistical significance at a p-value $<0.05$. We performed all statistical analyses using SPSS (version 23; IBM Corporation, Armonk, NY, USA).

\section{Results}

\section{Baseline characteristics of the study subjects}

We randomised $42(\mathrm{n}=22$ assigned to $M w$ arm; $\mathrm{n}=20$ assigned to placebo arm) subjects (figure 1). One patient each in the $M w$ arm and the placebo arm received a single dose and withdrew consent. Both these subjects were included in the primary and safety analysis.

The baseline parameters, the severity of illness, and comorbid illnesses in the two groups are described in table 1 . The study population was predominantly comprised of men (69\%) with a median (IQR) age of 50 (50-65) years. The median (IQR) time from symptom onset to randomisation was 7 (5-10) days and was similar in both the study arms. Fever, new-onset cough, and dyspnoea were frequently reported symptoms. More subjects in the placebo arm presented with dyspnoea and a lower platelet count. Any comorbid illness was seen in $57 \%(n=24)$ of subjects and $20 \%$ of them had more than one comorbid illness. Diabetes mellitus followed by systemic hypertension were common comorbid illnesses. The median (IQR) arterial oxygen tension/inspiratory oxygen fraction ratio was $186(135-234) \mathrm{mmHg}$ and was comparable in the two study arms. The median (IQR) baseline SOFA score was 3 (2-4). The median neutrophil/lymphocyte ratio was 7.5 and was not different between the $M w$ and the placebo arm. The baseline ordinal scale score was also similar in the two groups. Two-thirds of the study population required oxygen supplementation using a venturi-mask or a non-rebreather mask, while the remaining study subjects needed either high-flow nasal canula or mechanical ventilation at admission. All the subjects received systemic

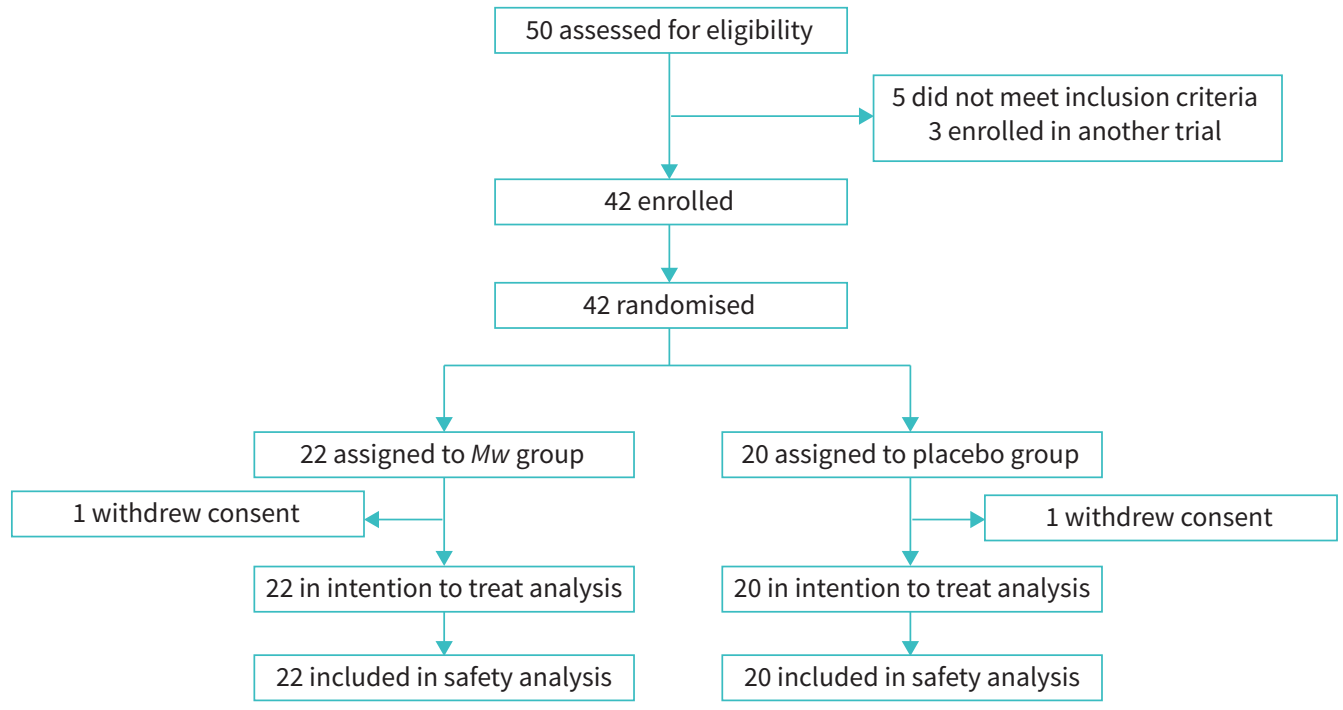

FIGURE 1 CONSORT diagram depicting the flow of subjects during the study. Mw: Mycobacterium w. 


\begin{tabular}{|c|c|c|c|c|}
\hline Parameter & Mw & Placebo & Total & p-value \\
\hline Subjects $\mathrm{n}$ & 22 & 20 & 42 & \\
\hline \multicolumn{5}{|l|}{ Demographics } \\
\hline Age years & 59 (52-62.5) & $51(45-65)$ & $56(50-65)$ & 0.16 \\
\hline Male & 13 (59) & $16(80)$ & 29 (69) & 0.19 \\
\hline \multicolumn{5}{|l|}{ Clinical parameters } \\
\hline $\begin{array}{l}\text { Time from symptom onset to } \\
\text { randomisation days }\end{array}$ & $9(5-14)$ & $7(4-10)$ & $7(5-10)$ & 0.16 \\
\hline \multicolumn{5}{|l|}{ Symptoms at presentation } \\
\hline Fever & 18 (81.8) & $18(90)$ & $36(85.7)$ & 0.61 \\
\hline Cough & 18 (81.8) & $14(70)$ & $32(76.2)$ & 0.44 \\
\hline Dyspnoea & 9 (40.9) & $15(75)$ & $24(57.1)$ & 0.03 \\
\hline $\begin{array}{l}\text { Presence of any comorbid } \\
\text { illness }\end{array}$ & $12(54.5)$ & $12(60)$ & $24(57.1)$ & 0.76 \\
\hline Hypertension & 6 (27.3) & 7 (35) & $13(31)$ & 0.74 \\
\hline Diabetes mellitus & $5(22.7)$ & $9(45)$ & 14 (33.3) & 0.19 \\
\hline COPD & $1(4.5)$ & $1(5)$ & $2(4.8)$ & 1.00 \\
\hline Asthma & $1(4.5)$ & $1(5)$ & $2(4.8)$ & 1.00 \\
\hline $\begin{array}{l}\text { Two or more comorbid } \\
\text { illnesses }\end{array}$ & $2(4.8)$ & $6(30)$ & $8(19)$ & 0.12 \\
\hline $\begin{array}{l}\text { Respiratory rate } \\
\text { breaths } \cdot \min ^{-1}\end{array}$ & $26(26-30)$ & $28(26-30)$ & $28(26-30)$ & 0.19 \\
\hline Heart rate beats $\cdot \min ^{-1}$ & $82(80-94)$ & $83(78-94)$ & $83(78-94)$ & 0.85 \\
\hline$P_{\mathrm{aO}_{2}} / F_{1 \mathrm{O}_{2}}$ ratio & $184(114-244)$ & 188 (152-225) & $186(135-234)$ & 0.68 \\
\hline Baseline SOFA score & $3(2-4)$ & $3(2-4)$ & $3(2-4)$ & 0.85 \\
\hline \multicolumn{5}{|l|}{ Investigations } \\
\hline Haemoglobin $\mathrm{g} \cdot \mathrm{dL}^{-1}$ & $12(11-13)$ & $12(11-13)$ & $12(1-13)$ & 0.33 \\
\hline Total leukocyte count $\mu \mathrm{L}$ & 9570 (7465-13033) & 8400 (5090-12215) & 8720 (5670-12523) & 0.23 \\
\hline Neutrophil/lymphocyte ratio & $7.6(6-13.5)$ & $6.7(4.7-17.7)$ & $7.5(5.3-13.7)$ & 0.66 \\
\hline Platelet count $\mu \mathrm{L}$ & $287(201-357)$ & 170 (127-309) & $226(156-323)$ & 0.03 \\
\hline Serum creatinine $\mathrm{mg} \cdot \mathrm{dL}^{-1}$ & $0.7(0.6-0.8)$ & $0.9(0.6-1.1)$ & $0.7(0.6-1)$ & 0.33 \\
\hline Serum albumin $\mathrm{g} \cdot \mathrm{dL}^{-1}$ & $3.5(3.3-3.7)$ & $3.5(3.2-3.9)$ & $3.5(3.2-3.8)$ & 0.61 \\
\hline Serum bilirubin $\mathrm{mg} \cdot \mathrm{dL}^{-1}$ & $0.5(0.4-0.8)$ & $0.5(0.4-0.6)$ & $0.5(0.4-0.6)$ & 0.44 \\
\hline ALT U. $L^{-1}$ & $39(29.3-85.3)$ & $42(26.5-77.7)$ & $39(28.8-78.5)$ & 0.92 \\
\hline AST $U \cdot L^{-1}$ & $45(30.5-61)$ & $46(37.8-58)$ & $45(32.8-55.6)$ & 0.61 \\
\hline $\mathrm{D}$-dimer $\mathrm{ng} \cdot \mathrm{mL}^{-1}$ & $603(254-840)$ & $621(245-1484)$ & $621(263-1145)$ & 0.58 \\
\hline $\begin{array}{l}\text { Score on the ordinal scale at } \\
\text { baseline }\end{array}$ & & & & 0.49 \\
\hline $\begin{array}{l}\text { 4: Hospitalised, requiring } \\
\text { oxygen supplementation }\end{array}$ & $14(63.6)$ & $15(75)$ & $29(69)$ & \\
\hline $\begin{array}{l}\text { 5: Hospitalised, requiring } \\
\text { high-flow oxygen devices } \\
\text { or NIV }\end{array}$ & 7 (31.8) & $5(25)$ & $12(28.6)$ & \\
\hline $\begin{array}{l}\text { 6: Hospitalised, receiving } \\
\text { invasive } \mathrm{MV} \text { or ECMO }\end{array}$ & $1(4.5)$ & 0 & $1(2.4)$ & \\
\hline \multicolumn{5}{|l|}{ Concomitant medication } \\
\hline Systemic glucocorticoids & $22(100)$ & $20(100)$ & $42(100)$ & \\
\hline Anticoagulation & $22(100)$ & $20(100)$ & $42(100)$ & \\
\hline Tocilizumab & $3(13.6)$ & $2(10)$ & $5(12)$ & 1.00 \\
\hline $\begin{array}{l}\text { Convalescent plasma } \\
\text { therapy }\end{array}$ & $2(9.1)$ & $1(5)$ & $3(7)$ & 1.00 \\
\hline Hydroxychloroquine & $4(18.2)$ & 7 (35) & $11(26.2)$ & 0.03 \\
\hline Antibiotics & $20(90.9)$ & $17(85)$ & $37(88.1)$ & 0.59 \\
\hline
\end{tabular}

Data are presented as median (first and third quartile) or $\mathrm{n}(\%)$, unless otherwise stated. Mw: Mycobacterium $w_{i} P_{\mathrm{aO}_{2}}$ : arterial oxygen tension; $F_{\mathrm{IO}_{2}}$ : inspiratory oxygen fraction; SOFA: sequential organ failure assessment score; ALT: alanine transaminase; AST: aspartate transaminase; NIV: noninvasive ventilation; MV: mechanical ventilation; ECMO: extracorporeal membrane oxygenation. 
glucocorticoids and anticoagulation. 11 subjects received hydroxychloroquine, while five subjects were treated with intravenous tocilizumab.

\section{Primary outcomes}

The odds of having a low ordinal scale score was significantly higher with the use of $M w$ on days 14 (OR 30.4 (95\% CI 3.3-276.4), p=0.002) and 21 (OR 14.9 (95\% CI 1.8-128.4), p=0.013), compared to the placebo arm (table 2). There was no difference in the clinical status between the two arms on day 28 . We did not find any difference in the delta SOFA score on days 7 and 14 between the two groups. Also, there was no difference in the maximum SOFA score during hospitalisation between the two study arms (table 2).

TABLE 2 Primary and secondary outcomes

\begin{tabular}{|c|c|c|c|c|}
\hline Parameter & Mw & Placebo & Total & p-value \\
\hline Subjects $n$ & 22 & 20 & 42 & \\
\hline \multicolumn{5}{|l|}{ Primary outcome } \\
\hline \multicolumn{5}{|l|}{ Clinical status (7-point scale) on day 14} \\
\hline 1: Not hospitalised with resumption of normal activities & $13(59.1)$ & $8(40)$ & $21(50)$ & \\
\hline \multicolumn{5}{|l|}{ 2: Not hospitalised, but unable to resume normal activities } \\
\hline 3: Hospitalised, not requiring supplemental oxygen & 2 (9) & $4(20)$ & $6(14.2)$ & \\
\hline 4: Hospitalised, requiring supplemental oxygen & $3(13.6)$ & $3(15)$ & $6(14.2)$ & \\
\hline $\begin{array}{l}\text { 5: Hospitalised, requiring nasal high-flow oxygen therapy, noninvasive } \\
\text { mechanical ventilation, or both }\end{array}$ & $1(4.5)$ & 0 & $1(2.4)$ & \\
\hline 6: Hospitalised, requiring ECMO, invasive mechanical ventilation, or both & $1(4.5)$ & $1(5)$ & $2(5)$ & \\
\hline 7: Death & $2(9)$ & $4(20)$ & $6(14.2)$ & \\
\hline Difference in clinical status distribution versus placebo, OR $(95 \% \mathrm{CI})^{\#}$ & $30.4(3.3-276.4)$ & Reference & & 0.002 \\
\hline \multicolumn{5}{|l|}{ Clinical status (7-point scale) on day 21} \\
\hline 1: Not hospitalised with resumption of normal activities & $16(72.7)$ & $11(55)$ & $27(64.3)$ & \\
\hline 2: Not hospitalised, but unable to resume normal activities & $1(4.5)$ & 0 & $1(2.4)$ & \\
\hline 3: Hospitalised, not requiring supplemental oxygen & 0 & $3(15)$ & $3(7.1)$ & \\
\hline 4: Hospitalised, requiring supplemental oxygen & $1(4.5)$ & 0 & $1(2.4)$ & \\
\hline $\begin{array}{l}\text { 5: Hospitalised, requiring nasal high-flow oxygen therapy, noninvasive } \\
\text { mechanical ventilation, or both }\end{array}$ & 0 & 1 (5) & $1(2.4)$ & \\
\hline $\begin{array}{l}\text { 6: Hospitalised, requiring ECMO (extracorporeal membrane oxygenation), } \\
\text { invasive mechanical ventilation, or both }\end{array}$ & $2(9.1)$ & $1(5)$ & $3(7.1)$ & \\
\hline 7: Death & $2(9.1)$ & $4(20)$ & $4(14.3)$ & \\
\hline Difference in clinical status distribution versus placebo, OR $(95 \% \mathrm{CI})^{\#}$ & $14.9(1.8-128.4)$ & Reference & & 0.013 \\
\hline \multicolumn{5}{|l|}{ Clinical status (7-point scale) on day 28} \\
\hline 1: Not hospitalised with resumption of normal activities & $16(72.7)$ & $14(70)$ & $30(71.4)$ & \\
\hline 2: Not hospitalised, but unable to resume normal activities & $1(4.5)$ & 0 & $1(2.4)$ & \\
\hline 3: Hospitalised, not requiring supplemental oxygen & 0 & $1(5)$ & $1(2.4)$ & \\
\hline 4: Hospitalised, requiring supplemental oxygen & $1(4.5)$ & 0 & $1(2.4)$ & \\
\hline $\begin{array}{l}\text { 5: Hospitalised, requiring nasal high-flow oxygen therapy, noninvasive } \\
\text { mechanical ventilation, or both }\end{array}$ & 0 & 0 & 0 & \\
\hline 6: Hospitalised, requiring ECMO, invasive mechanical ventilation, or both & 0 & 0 & 0 & \\
\hline 7: Death & $4(18.2)$ & $5(25)$ & $9(21.4)$ & \\
\hline Difference in clinical status distribution versus placebo, OR $(95 \% \mathrm{CI})^{\#}$ & $1.1(0.2-4.5)$ & Reference & & 0.95 \\
\hline Delta SOFA score at day 7 & $1(0-2.5)$ & $1(0-4)$ & $1(0-3)$ & 0.52 \\
\hline Delta SOFA score at day 14 & $0(0-0)$ & $0(0-1)$ & $0(0-0.8)$ & 0.35 \\
\hline Maximum SOFA score & $3(2-4)$ & $3(2-5)$ & $3(2-4)$ & 0.51 \\
\hline \multicolumn{5}{|l|}{ Secondary outcomes } \\
\hline 28-day mortality? & $4(18.1)$ & $5(25)$ & $9(21.4)$ & 0.69 \\
\hline Time to reduction by one-point on seven-point ordinal scale days & $9(5-10)$ & $7(3-10)$ & $7(4-10)$ & 0.52 \\
\hline Time to reduction by two-point on seven-point ordinal scale days & $12(11-14)$ & $11(8-24)$ & $12(10-15.3)$ & 0.85 \\
\hline Days on vasopressor drug $^{+}$days & $0.7(0.6-2.1)$ & $1(0.4-2.1)$ & $0.8(0.2-1.7)$ & 0.15 \\
\hline Days on mechanical ventilation ${ }^{+}$days & $2(2.5-6.5)$ & $4(1.3-9.3)$ & $3(1-6.2)$ & 0.83 \\
\hline ICU length of stay days & $8(4-11)$ & $8(4-13)$ & $8(4-12)$ & 0.84 \\
\hline Hospital length of stay days & $12(9.5-16)$ & $12(9-22)$ & $12(9.3-17.8)$ & 0.92 \\
\hline Time to PCR negativity days & $9(7-15.5)$ & $7.5(5-14)$ & $8.5(6.3-14)$ & 0.53 \\
\hline
\end{tabular}

Data are presented as median (first and third quartile) or $\mathrm{n}(\%)$, unless otherwise stated. Mw: Mycobacterium w; ECMO: extracorporeal membrane oxygenation; SOFA: sequential organ failure assessment score; ICU: intensive care unit. ${ }^{\#}$ : OR and $p$-value for the Mw treatment arm comparison were estimated using the proportional odds assumption after adjustment for baseline disease severity and use of experimental therapies; ": 28 -mortality calculated for 42 subjects lone patient in $M w$ arm withdrew consent after the first dose of the study drug and died on day 2, one patient in placebo arm left against medical advice and was assumed to have died); ${ }^{+}$: Mean (95\% $\mathrm{Cl}$ ). 


\section{Secondary outcomes}

One patient in the $M w$ and one in the placebo arm received a single dose and withdrew consent. Of these two, the subject who received $M w$ died after $48 \mathrm{~h}$ of hospitalisation, while the subject in the placebo arm went to another hospital and was presumed to have died. Finally, there were nine deaths (table 2). We did not find any difference in mortality in the two groups: four (18\%) died in the $M w$ arm versus five (25\%) in the placebo arm. We also did not observe any difference in the time to clinical improvement (a two-point improvement on an ordinal scale), days spent on a mechanical ventilator, days on vasopressor therapy, or time to achieve a negative RT-PCR (table 2). We did not find any difference in the ICU and the hospital length of stay between the two groups (table 2).

\section{Adverse events}

We found no safety concerns associated with the study drug, based on the assessments of organ dysfunction, vital signs, laboratory parameters, and local site reaction at the site of the injection.

\section{Discussion}

We found that $M w$ combined with standard care was safe and resulted in better clinical status on days 14 and 21 than those receiving routine care alone in critically ill patients with severe COVID-19. There was, however, no difference in the change in SOFA scores and mortality between the two groups.

Viral infection usually activates the intracellular pattern recognition receptors that result in effective viral clearance by type I and III interferons and leads to an effective adaptive immune response in most individuals [24]. However, COVID-19, like SARS evades innate immunity at multiple levels by impeding the production of type I and III interferons [7]. Also, those with the SARS-CoV-2 infection have impaired adaptive immune response manifested as peripheral lymphocytopenia $[2,4]$. There is also a reduction in the peripheral CD8 and CD4 T-cells in those with severe disease [5,6]. Furthermore, due to unabated viral replication, there is an activation of alveolar macrophages that causes a hyperinflammatory response in critically ill patients with SARS-CoV-2 $[1,2]$. Thus, there is both a state of hyper-immune response and an immune-suppressive state in SARS-CoV-2 [3].

$M w$ has been shown to induce apoptosis of activated macrophages by suppressing IL- $\beta$, thus subduing the hyperinflammatory response [25]. This is supported by our observation, where we could demonstrate a fall in inflammatory markers (like C-reactive protein) in patients with moderate-to-severe COVID-19 using $M w$ alone [26]. Also, $M w$ can potentially enhance viral clearance by its action via the MyD88 pathway of TLR-2 and TLR-4 activating Th1-mediated innate immune response [17, 27, 28]. This suggests $M w$ can modulate the immunity by suppressing the overexpressed inflammatory cytokines while at the same time inducing adaptive immune response for effective clearing of the virus.

We found an early resolution of respiratory failure in the $M w$ group compared to the placebo arm on days 14 and 21 but not day 28. This is because most patients had either improved or died by day 28, an observation similar to previous trials describing the use of remdesivir [29, 30]. However, unlike the remdesivir, the effect size was clinically significant in our study. We did not find any difference in the change in SOFA score or mortality between the two study arms due to the small sample size. Based on the results of this study, we are planning a larger trial. Importantly, similar to our previous experience with the use of $M w$, we found no adverse events attributable to $M w[19,26]$. The deaths were attributed to the progressive disease course of COVID-19 by investigators at each site.

Finally, our study is not without limitations. The small sample size makes the study underpowered to detect differences in mortality. We did not measure the cytokine levels in our subjects that could have enabled us to understand the mechanism of action of $M w$ in COVID-19. Future studies should also measure the Th1 and Th2 cytokines to elucidate the immune modulation by $M w$ in patients with COVID-19. The study is hypothesis generating. Based on the study's encouraging results, we have designed a larger study with mortality as the primary outcome.

In conclusion, the use of immunomodulator $M w$ in addition to standard care resulted in early clinical improvement compared to standard care alone. Larger multicentre trials are required to confirm our findings.

ARMY trial study group: Inderpaul Singh Sehgal (Dept of Pulmonary Medicine, Postgraduate Institute of Medical Education and Research, Chandigarh, India), Randeep Guleria (All India Institute of Medical Sciences, New Delhi, India), Sarman Singh (All India Institute of Medical Sciences, Bhopal, India), Mohammad Sabah Siddiqui (Dept of Medicine, All India Institute of Medical Sciences, Raipur, India), Anant Mohan (Dept of Pulmonary Medicine and Sleep Disorders, All India Institute of Medical Sciences, New Delhi, India), A. Jindal (All India Institute of Medical Sciences, Raipur, India), A. Bhalla (Dept of Internal Medicine, Postgraduate Institute of Medical Education and Research, Chandigarh, India), Kamal Kajal (Dept of Anesthesia, Postgraduate Institute of Medical Education and 
Research, Chandigarh, India), Pankaj Malhotra (Dept of Internal Medicine, Postgraduate Institute of Medical Education and Research, Chandigarh, India), Goverdhan Dutt Puri (Dept of Anesthesia, Postgraduate Institute of Medical Education and Research, Chandigarh, India), Sagar Khadanga (Dept of Internal Medicine, All India Institute of Medical Sciences, Bhopal, India), Rajnish Joshi (Dept of Internal Medicine, All India Institute of Medical Sciences, Bhopal, India), S. Saigal (Dept of Anaesthesia and Critical Care, All India Institute of Medical Sciences, Bhopal, India), Nitin M. Nagarkar (All India Institute of Medical Sciences, Raipur, India), Vikas Suri (Dept of Internal Medicine, Postgraduate Institute of Medical Education and Research, Chandigarh, India), Sushma Bhatnagar (Dept of Oncoanesthesia and Palliative Medicine, All India Institute of Medical Sciences, New Delhi, India), Pawan Tiwari (Dept of Pulmonary, Critical Care and Sleep Medicine, All India Institute of Medical Sciences, New Delhi, India), Mini P. Singh (Dept of Virology, Postgraduate Institute of Medical Education and Research, Chandigarh, India), Laxmi Narayana Yaddanapudi (Dept of Anesthesia, Postgraduate Institute of Medical Education and Research, Chandigarh, India), Saurabh Mittal (Dept of Pulmonary, Critical Care and Sleep Medicine, All India Institute of Medical Sciences, New Delhi, India) and Ritesh Agarwal (Dept of Pulmonary Medicine, Postgraduate Institute of Medical Education and Research, Chandigarh, India).

Conflict of interest: None declared.

Support statement: This study was supported by Council of Scientific and Industrial Research-New Millennium Indian Technology Leadership Initiative number 5/258/93/2020-NMITLI. Funding information for this article has been deposited with the Crossref Funder Registry.

\section{References}

1 McGonagle D, Sharif K, O’Regan A, et al. The role of cytokines including interleukin-6 in COVID-19 induced pneumonia and macrophage activation syndrome-like disease. Autoimmun Rev 2020; 19: 102537.

2 Domingo P, Mur I, Pomar V, et al. The four horsemen of a viral Apocalypse: the pathogenesis of SARS-CoV-2 infection (COVID-19). EBioMedicine 2020; 58: 102887.

3 Li H, Liu L, Zhang D, et al. SARS-CoV-2 and viral sepsis: observations and hypotheses. Lancet 2020; 395 : $1517-1520$.

4 Azkur AK, Akdis M, Azkur D, et al. Immune response to SARS-CoV-2 and mechanisms of immunopathological changes in COVID-19. Allergy 2020; 75: 1564-1581.

5 Wan S, Yi Q, Fan S, et al. Relationships among lymphocyte subsets, cytokines, and the pulmonary inflammation index in coronavirus (COVID-19) infected patients. Br J Haematol 2020; 189: 428-437.

6 Zhou F, Yu T, Du R, et al. Clinical course and risk factors for mortality of adult inpatients with COVID-19 in Wuhan, China: a retrospective cohort study. Lancet 2020; 395: 1054-1062.

7 Blanco-Melo D, Nilsson-Payant BE, Liu WC, et al. Imbalanced host response to SARS-CoV-2 drives development of COVID-19. Cell 2020; 181: 1036-1045.

8 Horby P, Lim WS, Emberson JR, et al. Dexamethasone in hospitalized patients with Covid-19 - preliminary report. $N$ Engl J Med 2020; 384: 693-704.

9 Sterne JAC, Murthy S, Diaz JV, et al. Association between administration of systemic corticosteroids and mortality among critically ill patients with COVID-19: a meta-analysis. JAMA 2020; 324: 1330-1341.

10 Akinosoglou K, Gogos C. Severe COVID-19 and interleukin-6 receptor antagonist tocilizumab: some notes of concern. Respirology 2020; 25: 1209.

11 Antinori S, Bonazzetti C, Gubertini G, et al. Tocilizumab for cytokine storm syndrome in COVID-19 pneumonia: an increased risk for candidemia? Autoimmun Rev 2020; 19: 102564.

12 Aziz M, Haghbin H, Abu Sitta E, et al. Efficacy of tocilizumab in COVID-19: a systematic review and meta-analysis. J Med Virol 2020; 93: 1620-1630.

13 Grifoni A, Weiskopf D, Ramirez SI, et al. Targets of T cell responses to SARS-CoV-2 coronavirus in humans with COVID-19 disease and unexposed individuals. Cell 2020; 181: 1489-1501.

14 Talwar GP, Zaheer SA, Mukherjee R, et al. Immunotherapeutic effects of a vaccine based on a saprophytic cultivable mycobacterium, Mycobacterium $w$ in multibacillary leprosy patients. Vaccine 1990; 8: 121-129.

15 Bezemer GFG, Garssen J. TLR9 and COVID-19: a multidisciplinary theory of a multifaceted therapeutic target. Front Pharmacol 2021; 11: 601685.

16 Singh IG, Mukherjee R, Talwar GP, et al. In vitro characterization of T cells from Mycobacterium w-vaccinated mice. Infect Immun 1992; 60: 257-263.

17 Adhikari A, Majumder S, Banerjee S, et al. Mycobacterium indicus pranii $(M w)$-mediated protection against visceral leishmaniasis: involvement of TLR4 signalling. J Antimicrob Chemother 2012; 67: 2892-2902.

18 Ahmad F, Mani J, Kumar P, et al. Activation of anti-tumor immune response and reduction of regulatory $\mathrm{T}$ cells with Mycobacterium indicus pranii (MIP) therapy in tumor bearing mice. PLoS One 2011; 6: e25424.

19 Sehgal IS, Agarwal R, Aggarwal AN, et al. A randomized trial of Mycobacterium $w$ in severe sepsis. J Crit Care 2015; 30: 85-89.

20 Sharma P, Misra RS, Kar HK, et al. Mycobacterium $w$ vaccine, a useful adjuvant to multidrug therapy in multibacillary leprosy: a report on hospital based immunotherapeutic clinical trials with a follow-up of 1-7 years after treatment. Lepr Rev 2000; 71: 179-192.

21 Sharma SK, Katoch K, Sarin R, et al. Efficacy and safety of Mycobacterium indicus pranii as an adjunct therapy in category II pulmonary tuberculosis in a randomized trial. Sci Rep 2017; 7: 3354

22 Mayosi BM, Ntsekhe M, Bosch J, et al. Prednisolone and Mycobacterium indicus pranii in tuberculous pericarditis. N Engl J Med 2014; 371: 1121-1130.

23 Kharkar R. Immune recovery in HIV with Mycobacterium W. J Indian Med Assoc 2002; 100: 578-579.

24 Newton AH, Cardani A, Braciale TJ. The host immune response in respiratory virus infection: balancing virus clearance and immunopathology. Semin Immunopathol 2016; 38: 471-482.

25 Pandey RK, Bhatt KH, Dahiya Y, et al. Mycobacterium indicus pranii supernatant induces apoptotic cell death in mouse peritoneal macrophages in vitro. PLoS One 2011; 6: e17093. 
26 Sehgal IS, Bhalla A, Puri GD, et al. Safety of an immunomodulator Mycobacterium w in COVID-19. Lung India 2020; 37: 279-281.

27 Pandey RK, Sodhi A, Biswas SK, et al. Mycobacterium indicus pranii mediates macrophage activation through TLR2 and NOD2 in a MyD88 dependent manner. Vaccine 2012; 30: 5748-5754.

28 Kumar P, Das G, Bhaskar S. Mycobacterium indicus pranii therapy induces tumor regression in MyD88- and TLR2-dependent manner. BMC Res Notes 2019; 12: 648.

29 Beigel JH, Tomashek KM, Dodd LE, et al. Remdesivir for the treatment of Covid-19 - preliminary report. $N$ Engl Med 2020; 383: 1813-1826.

30 Spinner CD, Gottlieb RL, Criner GJ, et al. Effect of remdesivir vs standard care on clinical status at 11 days in patients with moderate COVID-19: a randomized clinical trial. JAMA 2020; 324: 1048-1057. 